\title{
New records of long-legged flies (Diptera, Dolichopodidae) from Morocco
}

\author{
Mohamed Nourti ${ }^{1}$, Igor Ya. Grichanov², Kawtar Kettani ${ }^{1}$ \\ ${ }^{1}$ Faculté des Sciences, Université Abdelmalek Essaâdi \\ Tetouan, Morocco. E-mail: nourti.mohamed2@gmail.com \\ ${ }^{2}$ All-Russian Institute of Plant Protection \\ Podbelskogo 3, 196608, St.Petersburg-Pushkin, Russia.E-mail: grichanov@mail.ru
}

This paper provides new data on the distribution of 39 long-legged fly species (Diptera, Brachycera, Dolichopodidae) belonging to 19 genera from Morocco. Twelve species, Gymnopternus assimilis (Staeger, 1842), Hercostomus apollo (Loew, 1869), Hercostomus morenae (Strobl, 1899), Medetera media Parent, 1925, Medetera pallipes (Zetterstedt, 1843), Medetera petrophiloides Parent, 1925, Medetera aff. roghii Rampini et Canzoneri, 1979, Micromorphus minusculus Negrobov, 2000, Sciapus aff. negrobovi Naglis et Bartak, 2015, Campsicnemus simplicissimus Strobl, 1906, Sympycnus pulicarius (Fallén, 1823), and Syntormon pilitibia Grichanov, 2013 are newly added to the Moroccan insect fauna. In addition, available information on geographical distribution for each species is also included.

Key words: Dolichopodidae; Morocco; new records

\section{Introduction}

Dolichopodidae (Diptera, Brachycera) is a large, diverse and cosmopolitan family of flies commonly known as long-legged flies, including 8,000 species in 250 genera (Grichanov, 2017).

Morocco is part of the Mediterranean See Basin, lying between the Atlantic Ocean, the Mediterranean Sea and Sahara desert. Its territory can be conditionally divided into 7 natural areas: the North (Rif), East of Morocco (EM), Atlantic Plains (AP), Middle Atlas (MA), High Atlas (HA), Anti Atlas (AA) and Sahara (Rankou et al. 2015; Kettani, Negrobov 2016). Apart from the southeast regions (pre-Saharan and desert areas), Morocco's climate and geography are very similar to the Iberian Peninsula.

The Moroccan fauna of Dolichopodidae is insufficiently investigated and rarely found in literary sources as the distribution of individual species is comparatively rare.

The first contribution to the fauna of Dolichopodidae of Morocco was made in 1805 by Fabricius, who listed in his first checklist of Diptera of Morocco one species, Anahydrophorus cinereus (Fabricius, 1805) (as Scatophaga cinerea Fabricius, 1805). Later, several authors described or found new species either for science or for Morocco, as those of Macquart (1842), Becker, Stein (1913), Parent (1924-1950), Séguy (1930-1953), Vaillant (1950-1956), Boumezzough, Vaillant (1986), Gatt (2003), Pârvu (2006-2008), Grichanov (2009-2010), Grichanov, Negrobov (2014) and recently Ebejer et al., 2019.

As for the recent bibliographic data relating to exhaustive compilations on Dolichopodidae known in Morocco, three significant publications listed an inventory of these insects on the basis of literature records. One is that of Dakki (1997) who established a checklist of 32 species for aquatic Dolichopodidae within his contribution in the study of the national biodiversity dedicated to the fauna of Morocco. The earlier one was established by Negrobov (1991) who has included 44 species known from the country; however, some old species records were lost. The latest compilation was realized by Kettani and Negrobov (2016) who have compiled a check-list of Moroccan long-legged flies including 63 species; however, some old and recent species records were again lost. Later 16 species new for the country have been reported (Ebejer et al. 2019). Several species have been probably mentioned by various authors in error from the territory or recorded by females only. Their presence in Moroccan fauna must be confirmed.

Preparing a chapter for the forthcoming Catalog of Diptera of Morocco, we have found 90 species of the family published in many references since 1805. Nevertheless, the total number of Moroccan species can reach to 300 
species. For example, the fauna of neighbouring Spain contains nearly 200 and that of France - nearly 400 species (Naglis, Bartak, 2015; Grichanov, 2017).

Recent field work in different areas from Morocco has uncovered many species not previously known. The aim of this article is to contribute to a better knowledge of this fauna by providing new records for an up-to-date checklist.

\section{Material and methods}

This study was based on entomological field investigations to collect new samples from a multitude of habitats mainly belonging to the Rif and to a limited degree to the Atlasic domain, comprising a total of 53 surveyed sites.

The Rif, extending in an arc from the Straits of Gibraltar to the valley of Moulouya, are a succession of coastal mountains belonging to the Alpine system, which culminate somewhere near its center at Jbel Tidighine (2456 m). Table 1 summarizes the main geographical and cartographical data of studied sites. The altitudes of the stations range between 0 and $2017 \mathrm{~m}$. They cover a variety of habitats as forests, mountains, valleys, springs, streams, meadows, marshes, peat bogs, cultured fields and greenhouses, resulting from the diverse geomorphology and climate of the country.

The material referred to in this work comes from two main sources. The first part of the material presented in this work was captured by Nourti Mohamed between 2017 and 2019. The second part of the material is a large collection of specimens in alcohol, mostly hand swept from river banks and riparian vegetation by Kawtar Kettani and her students between 2010 and 2019. The material collected during joint field trips of all authors of this paper in July 2019 is also included. Two sites in the Rif Mountains have been visited (Figs 1, 2), the God's Bridge (or Pont de Dieu in French) at Akchour village (the Talassemtane National Park, 1 July) and the Lemtahane marsh at Amsemlil (the Bouhachem Natural Park, 4 July 2019). The specimens have been collected using entomological sweep nets (SN in the following list) and Malaise traps (MT), then transferred to bottles using aspirators or eclectors (Černý, von Tschirnhaus, 2014) and stored in $70 \%$ of alcohol.

Material cited in this work is housed at the Laboratoire Ecologie, Systématique et Conservation de la Biodiversité, Faculté des Sciences, Université Abdelmalek Essaâdi, Tétouan, Morocco and the Zoological Institute of the Russian Academy of Sciences, St. Petersburg, Russia.

\section{Results}

We founded 39 species belonging to 19 genera, including twelve species new for Morocco and two undescribed species. In the following list, the individual species are arranged alphabetically within each genus.

\section{Diaphorinae}

\section{Chrysotus Meigen, 1824}

\section{Chrysotus sp.}

MATERIAL. 60', 99, Rif, Douar El Hamma (forest), 35²3'05"N, 5³0'46"W, 338 m, 6-21.VI.2016 (MT), Coll. K. Kettani. NOTES. The material examined belongs to an undescribed species that will be described and illustrated separately.

\section{Chrysotus suavis Loew, 1857}

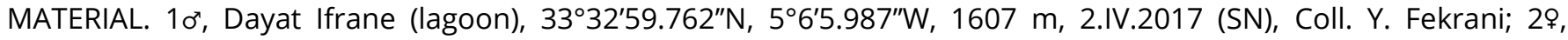
Tahanout (River), 31¹2'8.1"N, 7³3'55.749"W, 956 m, 25.III.2017 (SN), Coll. Y. Fekrani; 50', 6̊, Rif, Amsemlil env. (peat bog), 35¹5'614"N, 5²5'943"W, 1067 m, 4.VII.2019 (SN), Coll. M. Nourti.

REFERENCES. Grichanov, 2009, HA, Asni area (1100-1400 m)

TYPE LOCALITY: Germany: "Coln”; Austria: "Neusiedler See in Ungarn”.

DISTRIBUTION. Palaearctic: Afghanistan, Algeria, Armenia, Austria, Azerbaijan, Belgium, Bosnia and Herzegovina, Bulgaria, N China, Croatia, Czech Republic, Egypt, Estonia, Finland, France, Georgia, Germany, Greece (North Aegean), Hungary, Iraq, Iran, Israel, Italy, Kyrgyzstan, Latvia, Lithuania, Middle Asia, Mongolia, Morocco, Netherlands, Norway, Poland, Portugal, Romania, Russia (Adygea, Alania, Altai Rep., Astrakhan, Blagoveshchensk, Buryatia, Irkutsk, Kabardino-Balkaria, Kamchatka, Khabarovsk, Krasnodar, Krasnoyarsk, Kursk, Leningrad, Lipetsk, Magadan, Pskov, Rostov, Ryazan, Sakhalin, Tatarstan, Voronezh, Yakutia), Serbia, Slovakia, Spain (Canary Is), Sweden, Switzerland, Turkey (Adiyaman, Antalya, Antakya, Artvin, Gaziantep, Kizildere, Sanliurfa), UK, Ukraine (Cherkasy, Kherson, Odessa). 


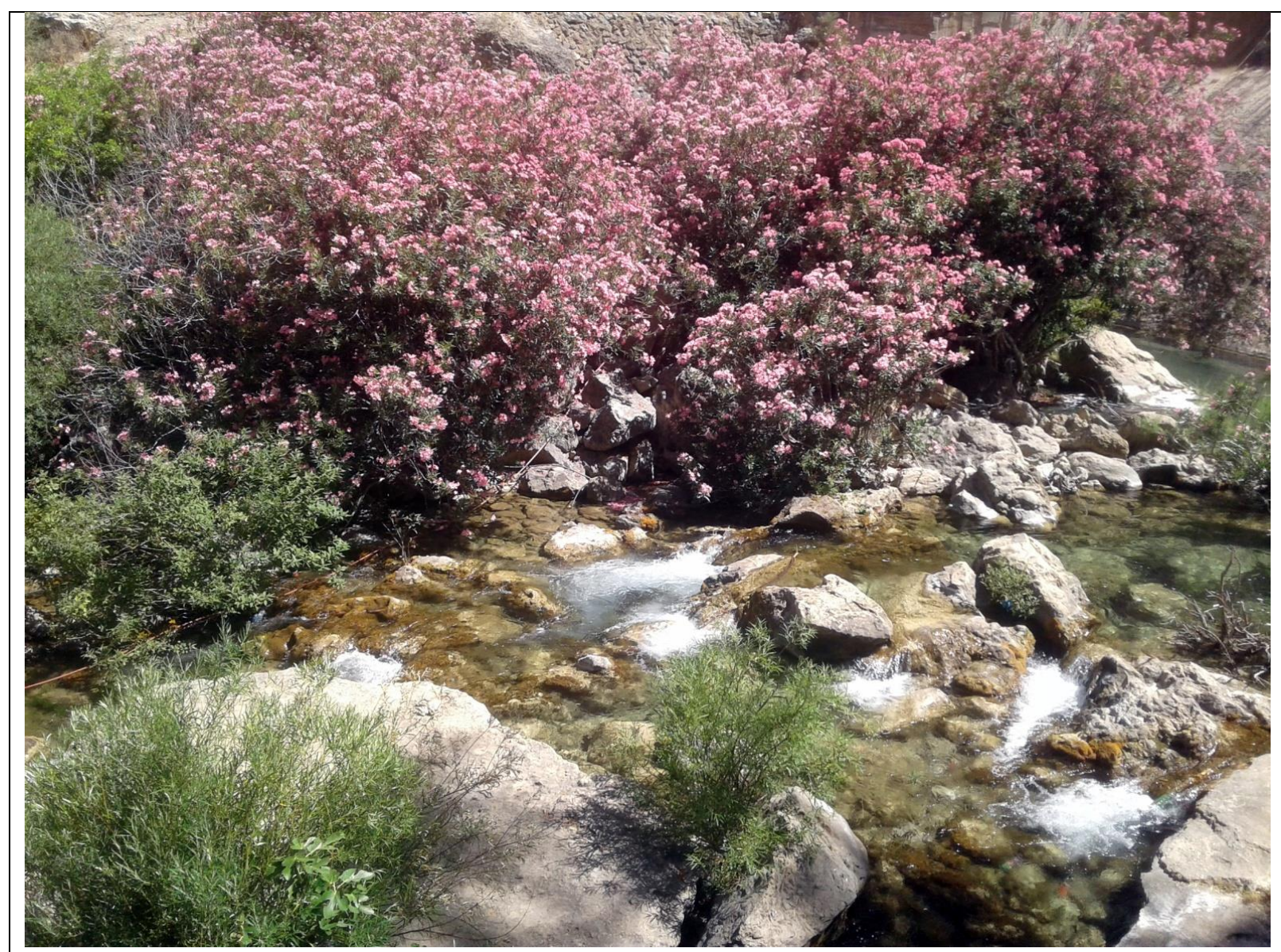

A

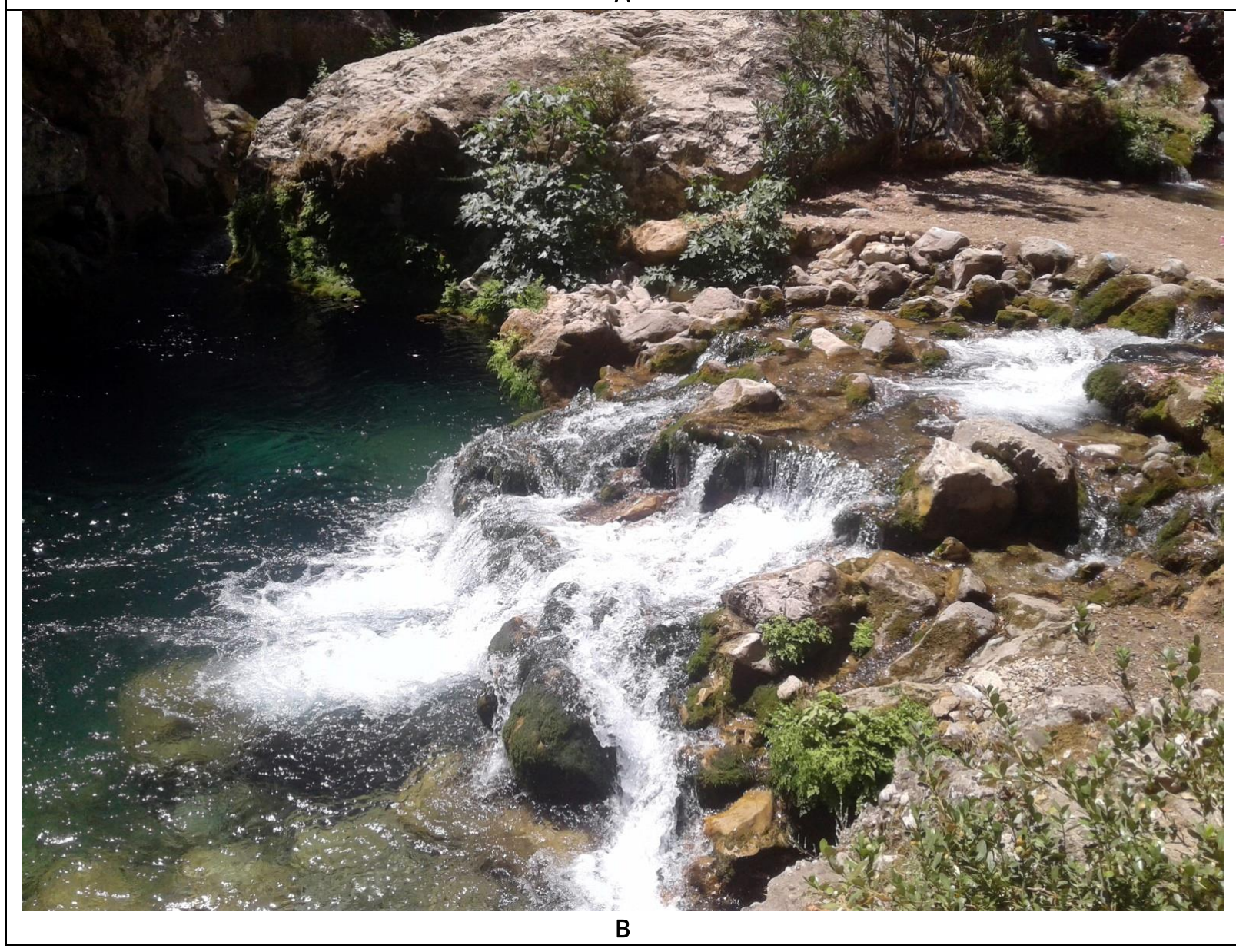

Fig. 1 (A-B). Oued Farda River at the God's Bridge, Talassemtane National Park, 1 July 2019 


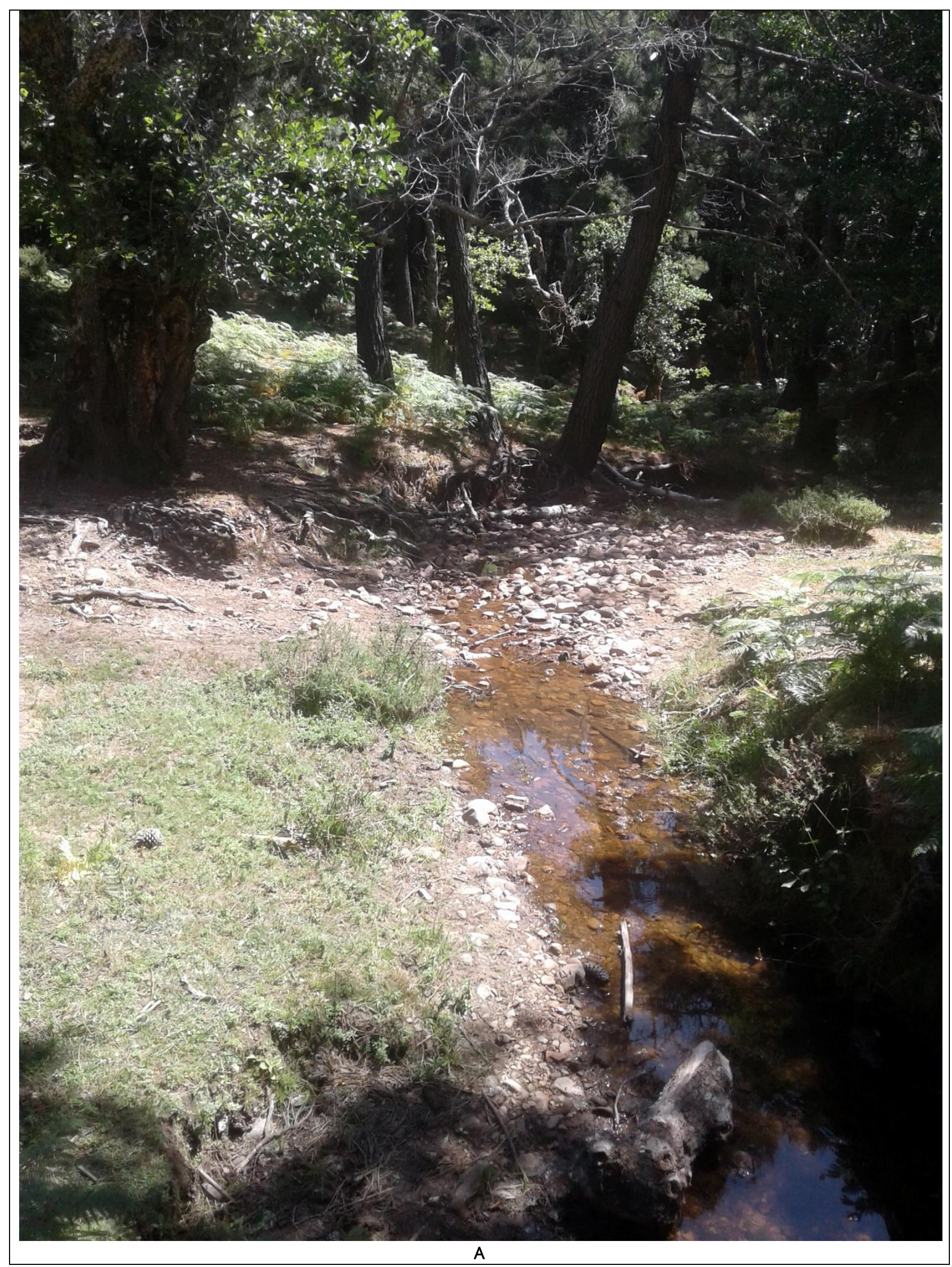




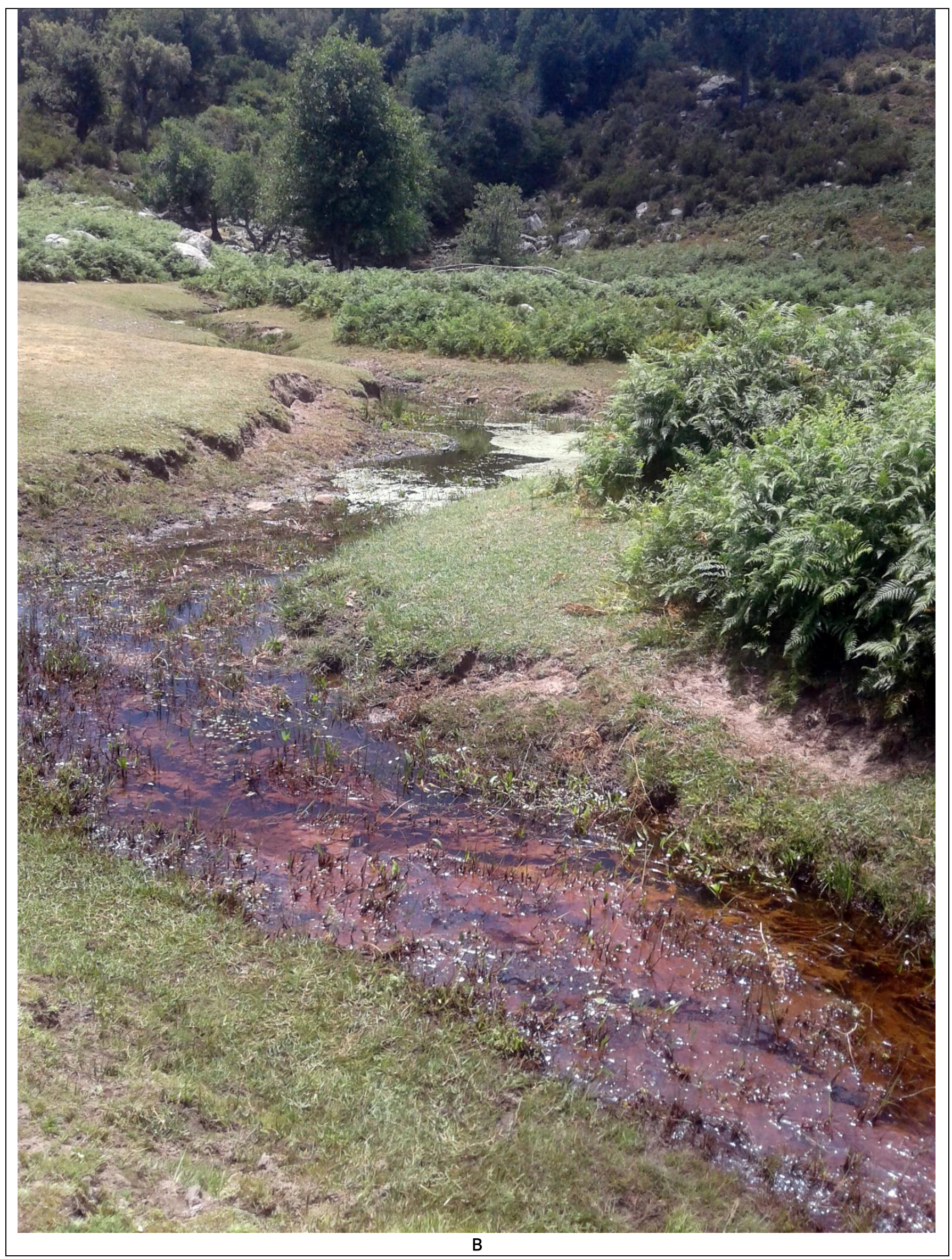

Fig. 2 (A-B). River Rehhal at the Lemtahane marsh, Bouhachem Natural Park, 4 July 2019 
Dolichopodinae

\section{Dolichopus Latreille, 1796}

\section{Dolichopus andalusiacus Strobl, 1899}

MATERIAL. 10', Rif, Plage Stihat (beach), 35²0'34.543"N, 457'4.018"W, 0 m, 26.IV-26.V.2015 (MT), Coll. K. Kettani. REFERENCES. Ebejer et al., 2019, AP, Loukkos marsh (2m).

TYPE LOCALITY. Spain: Algeciras.

DISTRIBUTION. Palaearctic: Algeria, France, Greece (Crete), Italy, Portugal, Spain, UK.

\section{Dolichopus griseipennis Stannius, 1831}

MATERIAL. 10", Adrou, PPNB [Project of Natural Park of Bouhachem] (forest), 35¹3'32.30"N, 5¹9'24.35"W, 556 m, 14.VII15.VIII.2013 (MT), Coll. K. Kettani.

REFERENCES. Parent, 1924, Rif, Tanger; Parent, 1927; Séguy, 1930, Rif, Tanger.

TYPE LOCALITY. France: Lyon.

DISTRIBUTION. Palaearctic: Algeria, ?Armenia, Azerbaijan, Austria, Belgium, Bulgaria, Cyprus, Czech Republic, Denmark, Egypt, Estonia, Finland, France, Georgia, Germany, Greece incl. Crete, Hungary, Iran, Ireland, Israel, Italy, N Kazakhstan, Lithuania, Luxembourg, Morocco, Netherlands, Norway, Poland, Portugal, Romania, Russia (Adygea, Krasnodar, Moscow, "Siberia"), Serbia, Slovakia, Spain incl. Balearic Is., Sweden, Switzerland, Tunisia, Turkey (Sinop), UK; “Middle Asia”.

\section{Dolichopus sabinus Haliday, 1838}

MATERIAL. 10`, Rif, Plage Stihat (beach), 35²0'34.543"N, 457'4.018"W, 4 m, 26.IV-26.V.2015 (MT), Coll. K. Kettani. REFERENCES. Ebejer et al., 2019, Rif, Martil (9m), Oued Laou (2m).

TYPE LOCALITY. Ireland: Killarney, Tarbert.

DISTRIBUTION. Palaearctic: Abkhazia, Austria, Azerbaijan, Belgium, Bulgaria, Czech Republic, Denmark, Estonia, Finland, France, Germany, Greece, Hungary, Ireland, Israel, Italy, Latvia, Netherlands, Norway, Poland, Portugal, Romania, Russia (Astrakhan, Kabardino-Balkaria, Saratov), Slovakia, Spain, Sweden, Turkey (Antalya), UK, Ukraine; Afrotropical: Tanzania.

\section{Gymnopternus Loew, 1857}

\section{Gymnopternus assimilis (Staeger, 1842)}

MATERIAL. 4 o', Rif, Amsemlil env. (peat bog), 35¹5'614"N, 5²5'943"W, 1067 m, 23.IV.2017 (SN), Coll. K. Kettani.

TYPE LOCALITY. Not given [Denmark].

DISTRIBUTION. Palaearctic: Austria, Belarus, Belgium, Czech Republic, Denmark, Estonia, Finland, France, Germany, Hungary, Ireland, the Netherlands, Poland, Russia (the Crimea, Karachai-Cherkessia, Leningrad, Moscow, Ryazan, Voronezh), Sweden, Switzerland, Turkey (Hakkari), the UK and the Ukraine (Kherson, Kyiv), Iran. First record for Morocco.

\section{Hercostomus Loew, 1857}

\section{Hercostomus apollo (Loew, 1869)}

MATERIAL. 20', 2\%, Rif, Chefchaouen, Talassemtane (Fir forest) 35²1'23"N, 5²6'55"W, 1696 m, 7.VI-17.X.2014 (MT), Coll. K. Kettani; $10^{\prime \prime}, 19$, Adrou, PPNB [Project of Natural Park of Bouhachem] (forest), 35¹3'32.30"N, 5¹9'24.35"W, 556 m, 14.VII15.VIII.2013, Coll. K. Kettani (MT); 20', 29, Rif, Amsemlil env. (peat bog), 35¹5'614"N, 5²5'943"W, 1067 m, $4 . \mathrm{VII} .2019$ (SN), Coll. M. Nourti.

TYPE LOCALITY. “Parnass, Griechenland” [Greece].

DISTRIBUTION. Palaearctic: Armenia, Greece, Iran, Iraq, Tunusia, Turkey (Taurus [Mountains]), Ukraine. First record for Morocco.

\section{Hercostomus aff. exarticulatoides Stackelberg, 1949}

MATERIAL. 20', 19, Rif, Akchour env., Pont de Dieu (river) 35¹3'42’N, 5¹0’31”W, 536 m, 1.VII.2019 (SN), Coll. M. Nourti. NOTES. The material examined belongs probably to an undescribed species that will be described and illustrated separately. At present, $H$. exarticulatoides is known only from the type locality in Tajikistan (Rakhati, Gissar ridge).

\section{Hercostomus excipiens Becker, 1907}

MATERIAL. 10", 1\%, Rif, Talembote (river) 35¹4'51"N; 5¹1'5"W, 440 m, 1.VI.2019 (SN), Coll. I. Grichanov. REFERENCES. Parent, 1924, Rif, Tétouan; Parent, 1927; Séguy, 1930, Rif, Oued Judios (Tanger).

TYPE LOCALITY. Algeria: “Umgebung von Algier und Zaghouan”.

DISTRIBUTION. Palaearctic: Spain, Algeria, Morocco. 
Hercostomus morenae (Strobl, 1899)

MATERIAL. 10', 49, Rif, Mnezla (marsh) 35³0'50,477"N, 548'18,374“W, 74 m, 3.I.2017 (SN), Coll. K. Kettani; $10^{\prime \prime}$, Chefchaouen,

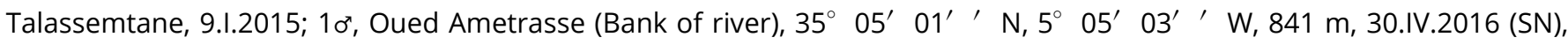
Coll. F.Z. Bahid \& A. Adghir; 30', 39, Bassin Tahaddart (dune marshland), 35³4'46.756"N, 559'15.832"W, 0m 31.I.2017 (SN), Coll. K. Kettani.

TYPE LOCALITY. Spain: Sierra Morena.

DISTRIBUTION. Palaearctic: Spain. First record for Morocco.

\section{Ortochile Berthold, 1827}

\section{Ortochile nigrocaerulea Latreille, 1779}

MATERIAL. 20', Rif, Douar El Hamma (forest), 35²3'05"N, 5³0'46"W, 338 m, 6.VI-21.VI.2016 (MT), Coll. K. Kettani; 20', 19,

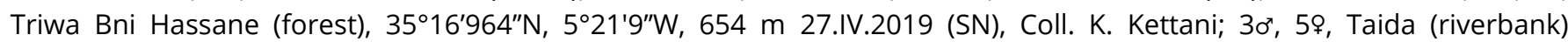
$35^{\circ} 21^{\prime} 12^{\prime \prime N}, 5^{\circ} 31^{\prime} 57^{\prime \prime W}, 501$ m, 25.IV.2015 (SN), Coll. K. Kettani.

REFERENCES. Parent, 1924, Rif, Tanger, Cap Spartel, Tétouan, Beni-Hozmar; Parent, 1927, Rif, Tanger; Séguy, 1930, Rif, Oued Judios (Tanger); Grichanov, 2009, Rif, Ouezzane (300m).

TYPE LOCALITY. France: Paris.

DISTRIBUTION. Palaearctic: Algeria, Austria, Bulgaria, Croatia, ?Denmark, France, Greece incl. North Aegean Is., Hungary, Israel, Italy, Morocco, Poland, Portugal, Spain incl. Balearic Is., Sweden, Syria, Tunisia, Turkey (Aydin, Izmir), UK.

\section{Poecilobothrus Mik, 1878}

Poecilobothrus appendiculatus (Loew, 1859), comb. nov.

=Hercostomus appendiculatus (Loew): Ebejer et al., 2019: 146

MATERIAL. 10', Rif, Perdicaris (park), 3547'26.675"N, 551'12.938"W, 223 m, 16.V.2015 (SN), Coll. K. Kettani.

REFERENCES. Parent, 1924, Rif, Tanger, Cap Spartel; Ebejer et al., 2019, Rif, Oued Nakhla (200m), Moulay Abdessalam (965m), Dardara (730m), Cap Spartel (155m).

TYPE LOCALITY. Spain: Ferrol.

DISTRIBUTION. Palaearctic: Algeria, Czech, France, Morocco, Portugal, Spain, Tunisia.

\section{Hydrophorinae}

\section{Hydrophorus Fallén, 1823}

\section{Hydrophorus balticus (Meigen, 1824)}

MATERIAL. 10`, MA, Mont Habri (forest), 33²1'36.55“N, 58'30.11“W, 2071 m, 2.IV.2017 (SN), Coll. Y. Fekrani.

REFERENCES. Vaillant 1956, HA, Djebel Toubkal, lac Tamhda (Anremer), Oukaimeden, Izourar; Boumezzough, Vaillant, 1986, HA, Djebel Toubkal (3100 m).

TYPE LOCALITY. Germany: Hamburg.

DISTRIBUTION. Palaearctic: Afghanistan, Algeria, Austria, Azerbaijan, Belgium, Bosnia and Herzegovina, Bulgaria, Cyprus, Czech Republic, Denmark, Estonia, Finland, France, Georgia, Germany, Greece, Hungary, Iran, Ireland, Israel, Italy, Mongolia, Morocco, Netherlands, Norway, Poland, Portugal, Romania, Russia (Adygea, Alania, Baikal, Chechnya, Kabardino-Balkaria, Karachai-Cherkessia, Krasnodar, Leningrad, Moscow, Voronezh, Yakutia), Slovakia, Spain, Sweden, Switzerland, Turkey (Antalya, Aydin, Denizli, Isparta, Kars, Mugla, Rize), UK, Ukraine; Afrotropical: South Africa, St. Helena.

\section{Liancalus Loew, 1857}

\section{Liancalus virens (Scopoli, 1763)}

MATERIAL. 10', Rif, Amsemlil env. (peat bog), 35¹5'614"N, 5²5'943"W, 1059 m, 23.IV.2017 (SN), Coll. K. Kettani.

REFERENCES. Vaillant, 1956, HA, Toubkal, Assif Tassouat (M'Goum), Aguelmous, Sidi Chamarouch, Imi N'Ifri; Boumezzough, Vaillant, 1986, HA, Tahanaout (750m), Adrar Anremer (2900m), AA, Djebel Siroua (3000m); Kettani \& Negrobov, 2016, AP, STifni - MNHNR (AP, S-Tifni).

TYPE LOCALITY. not given [“Carnioliae indigena", Slovenia].

DISTRIBUTION. Palaearctic: Abkhazia, Algeria, Austria, Azerbaijan, Belgium, Bulgaria, Cyprus, Czech, Denmark, Finland, France, Georgia; Germany, Golan Heights, Greece incl. Crete, Hungary, Iran, Ireland, Israel, West Bank, Italy, S Kazakhstan, Kyrgyzstan, Luxembourg, Morocco, Netherlands, Norway, Poland, Portugal incl. Madeira, Romania, Russia (Crimea, Krasnodar, Leningrad, “Siberia”), Slovakia, Slovenia, Spain, Sweden, Switzerland, Tajikistan, Tunisia, Turkey (Hakkari, Mugla), Turkmenistan, UK, Ukraine (Uzhgorod). 
MATERIAL. 20", Rif, Oued Souk Lhad (river bank), 35³'26"N, 54'10"W, 613 m, 30.IV.2016 (SN), leg. F.Z. Bahid \& A. Adghir; 10", Rif, Larache, Dayat Tazia (pond), 35²0'52"N, 5³3'12"W, 733 m, 23.IV.2017 (SN), Coll. K. Kettani.

REFERENCES. Parent, 1924, Rif, Cap Spartel, Tétouan; Parent, 1927; Pârvu et al. 2006, AP, Merja Zerga.

TYPE LOCALITY. England.

DISTRIBUTION. Palaearctic: Algeria, Belgium, Bulgaria, Denmark, France, Germany, Greece, Hungary, Ireland, Italy, Morocco, Netherlands, Poland, Portugal, Romania, Russia, Sweden, Tunisia, Turkey (Antalya, Erzurum), UK, Ukraine.

\section{Medeterinae}

\section{Medetera Fischer von Waldheim, 1819}

NOTES. Southern Palaearctic Medetera species are usually indistinguishable by females; therefore, some females collected are left unidentified.

\section{Medetera diadema (Linnaeus, 1767)}

MATERIAL. 10", Rif, Kitane (riverbank), 35³2'24.72"N, 5²0'23.58”W, 49 m, 4.VII.2015 (SN), Coll. K. Kettani; 10', Rif, Plage Stihat (beach), 35²0'34.543"'N, 457'4.018"'W, 26.IV-26.V.2015 (MT), Coll. K. Kettani.

TYPE LOCALITY. Germany: Lipsiae [= Leipzig].

REFERENCES. Parent, 1938 (no material provided); Séguy, 1953a (cf. diadema), AP, Rabat.

DISTRIBUTION: Palaearctic: Austria, Azerbaijan, Belarus, Belgium, Czech Republic, Denmark, Estonia, Finland, France, Georgia, Germany, Hungary, Iran, Ireland, Latvia, Moldova, Netherlands, Norway, Poland, Portugal, Romania, Russia (Krasnodar, Kursk, Krasnoyarsk, Leningrad, Lipetsk, Mordovia, Moscow, Pskov, Ryazan, Voronezh), Slovakia, Sweden, Switzerland, Turkey (Sultanice), UK, Ukraine.

\section{Medetera media Parent, 1925}

MATERIAL. 10', Rif, Tétouan, Faculty of Sciences (garden: on a tree trunk), 35³3'37"N, 5²1'51"W, 14 m, 3.VII.2019, Coll. I. Grichanov. TYPE LOCALITY. Tunisia.

DISTRIBUTION. Palaearctic: Egypt, Iran, Kazakhstan, Tunisia, Turkmenistan. First record for Morocco.

\section{Medetera micacea Loew, 1857}

MATERIAL. 10', HA, Imilchil, Lac Tislit (lagoon), 32¹9'44.33"N, 3²6'25.55"W, 2254 m, 29.VI.2017 (SN), Coll. Y. Fekrani \& N.H. El Ouazzani; 19, Rif, Ketama, Issaguen (forest), 3454'50.18"N, 4³4'41.35"W, 1547 m, 16.IV-16.V.2014 (MT), Coll. K. Kettani. REFERENCES. Ebejer et al., 2019, Rif, Dardara (730m).

TYPE LOCALITY. Not given [Europe].

DISTRIBUTION. Palaearctic: Austria, Belgium, Bosnia and Herzegovina, Bulgaria, China, Croatia, Denmark, Estonia, France, Germany, Greece, Hungary, Iran, Italy, Kazakhstan, Malta, Mongolia, Netherlands, Norway, Poland, Portugal, Romania, Russia (Adygea, Chechnya, Crimea, Krasnodar, Leningrad, Omsk, Voronezh, Yakutia), Slovakia, Spain, Sweden, UK, Ukraine (Cherkasy), Uzbekistan.

\section{Medetera pallipes (Zetterstedt, 1843)}

MATERIAL. 30', 39, Rif, Douar El Hamma (forest), 35²3'05"N, 5³0'46"W, 338 m, 6-21.VI.2016 (MT), Coll. K. Kettani. TYPE LOCALITY. Scania, “in Ostrog ad Wadstena; Botnia orientali ad Johannis Ro prope Tormea” [Sweden; Denmark]. DISTRIBUTION. Palaearctic: Austria, Belgium, Czech, Denmark, Egypt, Estonia, Finland, France, Georgia, Germany, Greece, Hungary, Iran, Israel, Morocco, Netherlands, Norway, Poland, Portugal, Romania, Russia (Adygea, Chechnya, Crimea, Kabardino-Balkaria, Karelia, Krasnodar, Leningrad, Novgorod, Pskov, Ryazan, Stavropol, Voronezh), Slovakia, Spain, Switzerland, Sweden, Turkey (Antalya, Hakkari, Kars), UK, Ukraine (Kherson). First record for Morocco.

\section{Medetera petrophiloides Parent, 1925}

MATERIAL. 10`, Rif, Ketama, Issaguen (forest), 3454'50.18"N, 4³4'41.35"W, 1547 m, 16.IV-16.V.2014 (MT), Coll. K. Kettani. TYPE LOCALITY. Belgium: Luc-S/Mer; France: Paris-Plage, Fermanville pres de Cherbourg.

DISTRIBUTION. Palaearctic: Belgium, Britain I., Bulgaria, Czech Republic, French mainland, Germany, Ireland, Portugal, South Russia, Slovakia, The Netherlands. First record for Morocco.

NOTES. Parent (1938) included Morocco into the distribution section for M. petrophila Kowarz, 1877 (no material was provided). It is very close to $M$. petrophiloides, differing reliably in fine structures of male genitalia. The presence of $M$. petrophila in Moroccan fauna must be confirmed.

\section{Medetera aff. roghii Rampini et Canzoneri, 1979}

MATERIAL. 10', Rif, Douar El Hamma (forest), 35²3'05"N, 5³0'46"W, 338 m, 6-21.VI.2016 (MT), Coll. K. Kettani.

TYPE LOCALITY. Spain: Menorca.

DISTRIBUTION. Palaearctic: Iran, Italy (Sicilia), Malta and Spain (Menorca). First record for Morocco. 


\section{Thrypticus Gerstäcker, 1864}

\section{Thrypticus bellus Loew, 1869}

MATERIAL. 10’, Rif, Jbel Bouhachem, Amsemlil env. (peat bog), 35¹5'614"N, 5²5'943"W, 1067 m, 4. VII.2019 (SN), Coll. I. Grichanov; 10", Rif, Tahaddart, Estuaire Oued Tahaddart (dune marshland), 35³4'46.756"N, 559'15.832"W, 0 m, 14. X.2017 $^{\circ}$ (SN), Coll. K. Kettani; 20', Rif, Tétouan, Barrage 9 Avril, 20.IV.2019 (SN), Coll. K. Kettani; 10", Rif, Plage Stihat (beach), 35²0'34.543"N, 457'4.018"W, 0 m, 26.IV-26.V.2015 (MT), Coll. K. Kettani.

REFERENCES. Séguy, 1930, EM, Dayat Sidi Kacem.

TYPE LOCALITY. England: Kew.

DISTRIBUTION. Afrotropical: DR Congo, Kenya, Ethiopia, Senegal, South Africa, St. Helena, Tanzania. Palaearctic: Abkhazia, Armenia, Austria, Azerbaijan, Belgium, Bosnia and Herzegovina, Bulgaria, China, Croatia, Czech, Denmark, Egypt, Finland, France, Germany, Greece, Hungary, Iran, Ireland, Israel, Golan Heights, Italy, Kazakhstan, Lithuania, Morocco, Netherlands, Poland, Portugal, Romania, Russia (Krasnoyarsk, Leningrad, Novosibirsk, Rostov, Vladivostok, Voronezh), Slovakia, Spain, Sweden, Switzerland, Turkey (Erzurum, Kars, Mugla), UK, Ukraine (Odessa).

\section{Neurigoninae}

\section{Neurigona Rondani, 1856}

\section{Neurigona solodovnikovi Grichanov, 2010}

=Neurigona punctifera Becker, 1907, in Kettani, Negrobov, 2016 (misidentification)

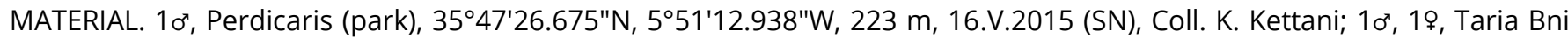
Faghloum (forest), $34.93175^{\circ} \mathrm{N}, 5.11541^{\circ} \mathrm{W}, 894 \mathrm{~m}, 11 . \mathrm{VV} .2019$ (MT), Coll. M. Ec-Charif; 7o', 19, Chrafate (riverbank), 3504'973"N, 506'567"W, 832 m, 2.V-16.VIII.2018 (MT), Coll. M. Nourti.

REFERENCES. Grichanov, 2010, AP, 40 km S Larache; Kettani, Negrobov, 2016, Rif, Ain Tissemlal (Azilane, 1255m).

TYPE LOCALITY. Morocco: $40 \mathrm{~km}$ S Larache.

DISTRIBUTION. Palaearctic: Morocco.

\section{Peloropeodinae}

\section{Chrysotimus Loew, 1857}

\section{Chrysotimus molliculoides Parent, 1937}

MATERIAL. 19, Rif, Larache, Dayat Tazia (pond), 35²0'52"N, 5³3'12"W, 733 m, 12.V.2015 (SN), Coll. K. Kettani. REFERENCES. Parent, 1937, MA, Ifrane (1600m).

TYPE LOCALITY. Morocco: Ifrane.

DISTRIBUTION. Palaearctic: Morocco.

\section{Micromorphus Mik, 1878}

\section{Micromorphus albipes (Zetterstedt, 1843)}

MATERIAL. 10", Rif, Oued Souk Lhad (river bank), 35³'26"N, 54'10"W, 613 m, 30.IV.2016 (SN), leg. F.Z. Bahid \& A. Adghir; 10", EM, Figuig, Bouanane (riverbank), 32²'5.05"N, 33'13.82"W, 855 m, 5.VI.2017 (SN), Coll. Y. Fekrani; 19, Rif, Tahaddart,

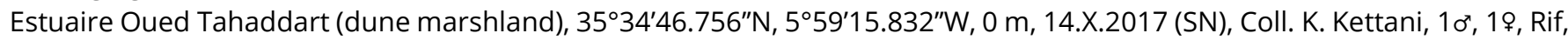
Tétouan, Barrage 9 Avril, 20.IV.2019 (SN), Coll. K. Kettani; 10', AA, Boudnib (desert), 3157'26.59"N, 3³4'39.74"W, 951 m, 3.IV.2017 (SN), Coll. Y. Fekrani; 10', Rif, Tétouan, Kharouba, 35³3'12"N, 5²1'19"W, 8.V.2018 (SN), Coll. M. Nourti; $10^{\circ}$, Rif,

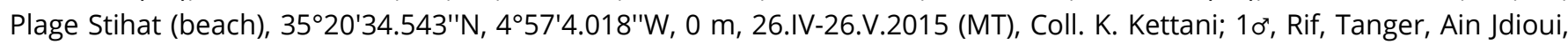
$35^{\circ} 34^{\prime} 12 " N, 5^{\circ} 55^{\prime} 12 " W, 20 . I V .2019$ (SN), Coll. N.E. El Ouazzani.

REFERENCES. Parent, 1924, Rif, Beni-Hozmar (Tétouan); Parent, 1927, Rif, Tétouan.

TYPE LOCALITY. Sweden: Ostrogoths; Larketorp.

DISTRIBUTION. Palaearctic: Algeria, Austria, Azores, Belgium, Bulgaria, China, Croatia, Czech, Denmark, Egypt, France, Germany, Greece, Hungary, Ireland, Iraq, Israel, Italy, Mongolia, Morocco, Netherlands, Poland, Romania, Russia (Krasnodar, Leningrad), Slovakia, Spain, Sweden, Switzerland, Turkey (Korucuk, Kizildere, Mugla), UK, Ukraine (Kherson, Odessa), West Bank; Oriental: Nepal; Australasian: New Zealand; Nearctic: USA: MD, SC, FL; MEX; Neotropical: Mexico, Costa Rica, Panama, Dominica.

NOTES. The material examined belongs to claripennis (Strobl, 1899) variety described from Spain (Algeciras).

\section{Micromorphus minusculus Negrobov, 2000}

MATERIAL. 10’, EM, Figuig, Bouanane (riverbank), 32²'5.05"N, 3³'13.82"W, 855 m, 5.IV.2017 (SN), Coll. Y. Fekrani; 10, AA, Taroudant, Taliouine (agricultural field), 30³1'28.847"N, 755'14.779"W, 1014 m, 13.VIII.2017 (SN), Coll. Y. Fekrani. 
TYPE LOCALITY. Ukraine: Odessa Prov., “Belgrad, Japug Lake” [=Bolgrad, Yalpug Lake].

DISTRIBUTION. Palaearctic: Tajikistan, Ukraine (Odessa). First record for Morocco.

\section{Rhaphiinae}

\section{Rhaphium Meigen, 1803}

\section{Rhaphium brevicorne Curtis, 1835}

$=$ Xiphandrium pectinatum Becker, in Vaillant, 1956

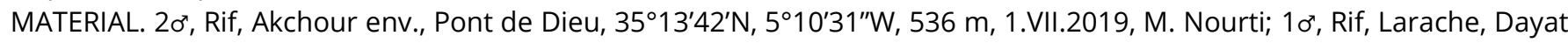

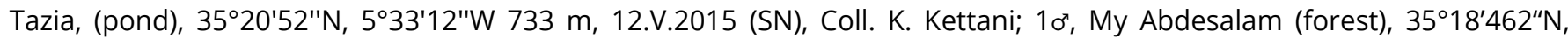
5³1'10.231“W, 1177 m, 26.VI.2017 (SN), Coll. K. Kettani.

REFERENCES. Vaillant, 1956, AP, Rabat, HA, Oukaimeden; Kettani, Negrobov, 2016, AP, Rabat.

TYPE LOCALITY. England: Isle of Wight.

DISTRIBUTION. Palaearctic: Algeria, Belgium, France incl. Corsica, Germany, Greece (North Aegean), Iraq, Ireland, Israel, Italy, Lebanon, Morocco, Netherlands, Portugal, Russia (Krasnodar), Spain incl. Canary Is., Sweden, Tajikistan, Turkey (Kizildere), UK.

\section{Rhaphium caliginosum Meigen, 1824}

= Raphium lanceolatum Loew, 1850, in Kazerani et al., 2013

MATERIAL. 10', Rif, Amsemlil env. (peat bog), 35¹5'614"N, 5²5'943"W, 1067 m, 4.VII.2019 (SN), Coll. M. Nourti; 10", Chefchaouen, Bni selmane, Dbani (meadow), 354'0.625"N, 50'34.282"W, 1046 m, 19.V.2018 (SN), Coll. M. Nourti.

REFERENCES. Parent, 1924, Rif, Cap Spartel; Kazerani et al. 2013 (no material provided).

TYPE LOCALITY. not given.

DISTRIBUTION. Palaearctic: Algeria, Armenia, Austria, Azerbaijan, Bosnia and Herzegovina, Bulgaria, Denmark, Estonia, Finland, France, Germany, Greece, Israel, Golan Heights, Italy, Kazakhstan, Latvia, Morocco, Netherlands, Norway, Portugal, Romania, Russia (Adygea, Alania, Kabardino-Balkaria, Kaliningrad, Karachai-Cherkessia, Krasnodar, Krasnoyarsk, Kursk, Leningrad, Moscow, Murmansk, Pskov, Rostov, Stavropol, Tatarstan, Voronezh), Serbia, Sweden, Switzerland, Syria, Turkey (Burdur, Izmir), UK, Ukraine (Kherson, Odessa).

\section{Sciapodinae}

\section{Sciapus Zeller, 1842}

\section{Sciapus aff. negrobovi Naglis et Bartak, 2015}

MATERIAL. 7o', Rif, Plage Stihat (beach), 35²0'34.543"N, 457'4.018"W, 0 m, 26.IV-26.V.2015 (MT), Coll. K. Kettani; $10^{\circ}$, Rif, Kitane (riverbank), 35³2'24.72"N, 5²0'23.58”W, 49 m, 4.VII.2015 (SN), Coll. K. Kettani.

TYPE LOCALITY. Portugal, Valhelhas.

DISTRIBUTION. Palaearctic: Portugal. First record for Morocco.

NOTES. Hypopygium morphology is practically identical to Fig. 3 (Naglis et Bartak, 2015). Some fine differences we consider relating to individual variability or inaccuracy of the description. E.g., length ratio of fore tibia and basitarsus varies in our material. Male postabdomen is dark in our specimens. Hypopygium of Sciapus negrobovi was described as mainly yellow; however, this colour is almost impossible, if the abdomen is "metallic green, with tergites 2-5 with large yellow dorsolateral patch". CuAx ratio is 1.1 in Sciapus negrobovi, but 0.5-0.7 in our specimens.

\section{Sympycninae}

\section{Campsicnemus Haliday in Walker, 1851}

\section{Campsicnemus simplicissimus Strobl, 1906}

MATERIAL. 20', Rif, Plage Stihat (beach), 35²0'34.543"N, 457'4.018"W, 26.IV-26.V.2015 (MT), Coll. K. Kettani. TYPE LOCALITY: Spain: Algeciras.

DISTRIBUTION. Palaearctic: Abkhazia, Bulgaria, France, Greece (North Aegean), Hungary, Italy, Israel, Portugal, Russia (Astrakhan, Karachai-Cherkessia, Krasnodar, Rostov), Spain, Switzerland, Tajikistan, Turkey (Antalya, Beysehir lake). First record for Morocco.

\section{Sympycnus Loew, 1857}


DISTRIBUTION. Nearctic: USA: California; Palaearctic: Andorra, Austria, Azerbaijan, Belgium, Bosnia and Herzegovina, Bulgaria, Croatia, Czech, Denmark, Estonia, Finland, France, Georgia, Germany, Greece, Hungary, Iran, Ireland, Italy, Kazakhstan, Kyrgyzstan, Latvia, Lithuania, Luxembourg, Macedonia, Moldova, Mongolia, Netherlands, Norway, Poland, Portugal, Romania, Russia (Alania, Altai Rep., Altai Ter., Buryatia, Chechnya, Chelyabinsk, Crimea, Kabardino-Balkaria, Kaliningrad, Karachai-Cherkessia, Karelia, Khakassia, Krasnodar, Krasnoyarsk, Leningrad, Lipetsk, Moscow, Murmansk, Novgorod, Novosibirsk, Pskov, Stavropol, Svedlovsk, Tambov, Tatarstan, Voronezh, Yakutia), Serbia, Slovakia, Spain incl. Canary Islands, Sweden, Switzerland, Tajikistan, Turkey (Aydin, Bolu, Mugla, Van), UK, Ukraine (Kherson), Uzbekistan. First record for Morocco.

\section{Syntormon Loew, 1857}

NOTES. Southern Palaearctic Syntormon species are usually indistinguishable by females; therefore, some females collected are left unidentified.

\section{Syntormon denticulatus (Zetterstedt, 1843)}

=Syntormon pumilus Parent, 1925 (nec Meigen, 1824; misidentification), in Pârvu et al., 2006

MATERIAL. 10', Rif, Plage Stihat (beach), 35²0'34.543"N, 457'4.018'W,4m 26.IV-26.VI.2016 (MT), Coll. K. Kettani; $10^{\circ}$, Amelay (meadow), 35¹1'44"N, 5¹8'36"W 294 m, 2.V.2018, (SN), Coll. M. Nourti; 10', Taliouine, (agricultural field), 30³1'28.847"N, 755'14.779"W 1014 m. 2.IX.2017 (SN), Coll. Y. Fekrani; 10’, Tétouan, M'Diq, Koudiat Taifour (forest), 3540'28.706N, 5¹9'1.841"W, 100 m, 31.V-14.VI.2018 (MT), Coll. M. Nourti; 10', Rif, Amsemlil env. (peat bog), 35¹5'614"N, 5²5'943"W, 1067 m, 4.VII.2019 (SN), Coll. M. Nourti; Rif, Larache, Dayat Tazia, 35²0'52"N, 5³3'12'"W, 733 m, 23.IV.2017; 10', Rif, Oued Souk Lhad (river bank), 35³'26"N, 54'10"W, 613 m, 30.IV.2016 (SN), leg. F.Z. Bahid \& A. Adghir; 10", Rif, Amsemlil env. (peat bog), 3515'614"N, 5²5'943"W, 1067 m, 23. IV. 2017 (SN), Coll. K. Kettani; 10', Taliouine (agricultural field), 30³1'28.847"N, 755'14.779"W, 1014 m, 2.IX.2017 (SN), Coll. Y. Fekrani; 10", HA, Sidi Masali, Ain Taferaout (spring), 31¹3'56,53"N, 758'53,59"W, 1237 m, 31.XII.2017 (SN), Coll. Y. Fekrani.

REFERENCES. Parent, 1924, Rif, Tétouan; Parent, 1927, Rif, Tétouan; Séguy, 1930, Rif, Tanger; Pârvu et al. 2006, AA, Lac Edehby, Ouarzazate.

TYPE LOCALITY. Sweden: Scania.

DISTRIBUTION. Palaearctic: Abkhazia, Afghanistan, Armenia, Azerbaijan, Belarus, Bulgaria, Estonia, Finland, France, Germany, Greece, Iran, Israel, Italy, Kyrgyzstan, Morocco, Norway, Poland, Portugal, Romania, Russia (Adygea, Alania, Chechnya, Kabardino-Balkaria, Karelia, Leningrad, Moscow, Murmansk, Stavropol), Sweden, Switzerland, Tajikistan, Turkey (Erzurum, Muğla, Van), UK, Ukraine.

\section{Syntormon mikiStrobl, 1899}

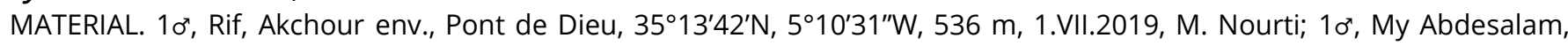

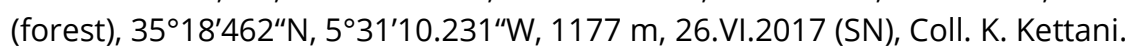

REFERENCES. Parent, 1927, “Maroc”.

TYPE LOCALITY. Spain: Algeciras.

DISTRIBUTION. Palaearctic: Bulgaria, Czech, France incl. Corsica, Greece incl. Crete, Ireland, ?Israel, Italy, Morocco, Poland, Portugal, Spain, "Yugoslavia", Sweden, Tunisia, UK.

\section{Syntormon pallipes (Fabricius, 1794)}

MATERIAL. 3, Rif, Oued Souk Lhad (river bank), 35³'26"N, 54'10"W, 613 m, 30.IV.2016 (SN), leg. F.Z. Bahid \& A. Adghir; 20', Chefchaouen, Talassemtane (Forest), 35 $15^{\prime} 19.648^{\prime}$ ' N, $5^{\circ} 19^{\prime} 7.521^{\prime}$ ' W, 339 m 9.I.2015 (SN), Coll. K. Kettani; 19 , Perdicaris (park), 3547'26.675"N, 551'12.938"W, 223 m, 16.V.2015 (SN), Coll. K. Kettani; 140', 9\%, Rif, Amsemlil env. (peat bog), 35¹5'614"N, 5²5'943"W, 1067 m, 4.VII.2019 (SN), Coll. M. Nourti.

NOTES. Both pallipes and pseudospicatus phenotypes are present in Morocco.

REFERENCES. Parent, 1924, Rif, Cap Spartel, Tétouan, Xauen; Parent, 1927, Rif, Cap Spartel.

TYPE LOCALITY. Germany.

DISTRIBUTION. Palaearctic: Abkhazia, Afghanistan, Algeria, Armenia, Austria, Azerbaijan, Belgium, Bosnia and Herzegovina, Bulgaria, China, Croatia, Czech, Denmark, Egypt, Estonia, Finland, France, Georgia, Germany, Greece incl. Crete, Hungary, Iceland, Iran, Iraq, Ireland, Israel, Italy, Jordan, Kyrgyzstan, Latvia, Morocco, Netherlands, Norway, Poland, Portugal incl. Madeira, Azores, Romania, Russia (Adygea, Alania, Chechnya, Crimea, Kabardino-Balkaria, Karachai-Cherkessia, Krasnodar, Leningrad, Murmansk, Rostov, Voronezh), Serbia, Slovakia, Spain, Sweden, Switzerland, Tajikistan, Tunisia, Turkey (Adiyaman, Ankara, Antalya, Burdur, Denizli, Hakkari, Isparta, Korucuk, Mugla, Van), UK, Ukraine (Kherson, Odessa), Uzbekistan; Oriental: China; Afrotropical: Madagascar, Tanzania, Yemen, St Helena (?introduced).

\section{Syntormon pilitibia Grichanov, 2013}

MATERIAL. 10’, Rif, Amsemlil env. (peat bog), 35¹5'614"N, 5²5'943"W, 1067 m, 4.VII.2019 (SN), Coll. M. Nourti.

TYPE LOCALITY. Israel: Menahamiya.

DISTRIBUTION. Palaearctic: Israel. First record for Morocco. 


\section{Syntormon zelleri(Loew, 1850)}

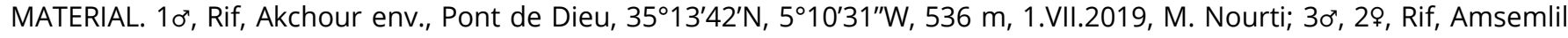
env. (peat bog), 35¹5'614"N, 5²5'943"W, 1067 m, 4.VII.2019 (SN), Coll. M. Nourti; 10', Rif, Amsemlil env. (peat bog), 35¹5'614"N, 5²5'943"W, 1059 m, 23.IV.2017 (SN), Coll. K. Kettani.

REFERENCES. Vaillant, 1956, HA, Oukaimeden, Izourar; Pârvu et al. 2006, AP, Merja Zerga.

TYPE LOCALITY. Italy: "Sicilien".

DISTRIBUTION. Palaearctic: Abkhazia, Austria, Azerbaijan, Belgium, Czech, France, Germany, Greece incl. Crete, Hungary, Iran, Ireland, Italy, Netherlands, Portugal, Romania, Russia (Chechnya, Krasnodar), Slovakia, Spain, Switzerland, Turkey (Afyonkarahisar, Usak), UK, "Middle Asia".

\section{Teuchophorus Loew, 1857}

\section{Teuchophorussp.}

MATERIAL. 10", Rif, Chefchaouen, Oued Souk Lhad (river bank), 35.057222 N, 5.069444 W, 820 m, 30. IV.2016 (SN), leg. F.Z. Bahid \& A. Adghir.

NOTES. The material examined belongs to an undescribed species that will be described and illustrated separately.

\section{Acknowledgements}

The work of IYG is performed within the Program for Basic Scientific Researches in the Russian Federation for the LongTerm Period (2013-2020) approved by Order No. 2538-r of the Government of the Russian Federation dated 27 December 2012, and supported by the All-Russian Institute of Plant Protection project N 0665-2019-0014.

\section{References:}

Becker, Th. \& Stein, P. (1913). Dipteren aus Marokko. Annuaire du Musée zoologique de l'Académie des sciences de St. Pétersbourg, 18, 62-95.

Boumezzough, A. \& Vaillant, F. (1986). Les Diptères Dolichopodidae Hydrophorinae du Maroc. L'Entomologiste, 42, 5, 295300.

Černý, M. \& von Tschirnhaus, M. (2014). New records of Agromyzidae (Diptera) from the Afrotropical Region, with a checklist. Acta Musei Silesiae, Scientiae Naturales, 63, 159-176.

Dakki, M. (1997). Etude Nationale sur la Biodiversité, Faune Aquatique Continentale. Rapport PNUE \& Ministère d'environnement, Maroc.

Ebejer, M.J., Kettani, K. \& Gatt, P. (2019). First records of families and species of Diptera (Insecta) from Morocco. Boletín de la Sociedad Entomologica Aragonesa, 64, 143-153.

Fabricius, J.C. (1805). Systema antliatorum secundum ordines, genera, species adiectis synonymis, locis, observationibus, descriptionibus. Brunsvigae [= Brunswick]: Apud Carolum Reichard, 372.

Gatt, P. (2003). New species and records of Microphorella Becker (Diptera: Empidoidea, Dolichopodidae) from the Mediterranean region. Revue suisse de zoologie, 110, 669-684.

Grichanov, I.Ya. (2009). New records for Mediterranean Dolichopodidae (Diptera). An International Journal of Dipterological Research, 20, 4, 207-215.

Grichanov, I.Ya. (2010). West-Palearctic species of the genus Neurigona Rondani (Diptera: Dolichopodidae). Russian Entomological Journal, 19, 3, 249-256.

Grichanov, I.Ya. (2017). Alphabetic list of generic and specific names of predatory flies of the epifamily Dolichopodoidae (Diptera). 2nd Edition. St.Petersburg: VIZR, 1-563. (Plant Protection News Supplements, N23). Available from: https://archive.org/download/Grichanov2017DolibankSec/Grichanov\%202017\%20Dolibank-sec.pdf/ (accessed 15 October 2018).

Grichanov, I.Ya. \& Negrobov, O.P. (2014). Palaearctic species of the genus Sciapus Zeller (Diptera: Dolichopodidae). St. Petersburg: VIZR (Plant Protection News Supplements, 13).

Kazerani, F., Khaghaninia, S., Talebi, A.A. \& Grichanov, I.Ya. (2014). Faunistic survey of Dolichopodidae in forests of northern Iran with nine species as new records for the country. Zoology and Ecology, 24, 3, $266-273$. DOI:10.1080/21658005.2014.937926

Kettani, K. \& Negrobov, O. (2016). The updated check list of Dolichopodidae of Morocco (Diptera). Cesa News, $128,1-8$.

Macquart, J. (1842). Diptères exotiques nouveaux ou peu connus. Tome deuxième. 2e partie. Mémoires de la Société des sciences, de l'agriculture et des arts de Lille, 1841, 1, 65-200.

Naglis, S. \& Bartak, (2015). Dolichopodidae (Diptera) from the Iberian Peninsula, with description of three new species. Zootaxa, 3964, 1, 125-137.

Negrobov, O.P. (1991). Dolichopodidae. In: Soos, A., Papp, L. \& Oosterbroeck, P. (Eds.). Catalogue of Palaearctic Diptera 7: Dolichopodidae-Platypezidae. Budapest: Akadémiai Kiadó, 1-291. DOI: 10.1016/B978-0-444-98731-0.50008-9.

Parent, O. (1924). Deux dolichopodidés nouveaux capturés au Maroc espagnol. Publ Junta Ciències Naturals de Barcelone, $4,6,3-15$. 
Parent, O. (1927). Contribution à l'étude de la distribution géographique de quelques espèces de Dolichopodides. Comptesrendus du Congrès des sociétés savantes en 1926. Science, 449-484.

Parent, O. (1937). Diptères Dolichopodides nouveaux du Congo belge et du Maroc. Bulletin du Musée Royal d'Histoire Naturelle de Belgique. Bruxelles, 13, 18, 1-19.

Parent, O. (1938). Diptères Dolichopodidae. Faune de France, 35, 1-720.

Pârvu, C., Mirceni, R.P. \& Zaharia, R. (2006). Faunistic data on some dipterian families (Insecta, Diptera) from Morocco (Results of «Hamada» Expedition 2005). Travaux du Muséum National d'Histoire Naturelle «Grigore Antipa», 49, $271-281$.

Rankou, H., Culham, A., Sghir Taleb, M., Ouhammou, A., Martin, G. \& Jury, S.L. (2015). Conservation assessments and Red Listing of the endemic Moroccan flora (monocotyledons). Botanical Journal of the Linnean Society, 177, 4, 504-575. doi: https://doi.org/10.1111/boj.12258.

Séguy, E. (1930). Contribution à l'étude des Diptères du Maroc. Mémoires de la Société des Sciences Naturelles du Maroc, $24,206$.

Seguy, E. (1953). Diptères du Maroc. Encyclopédie entomologique. Série B, Mémoires et notes (1947-1953), 11, 77-92.

Vaillant, F. (1956). Recherches sur la faune madicole de France, de Corse et d'Afrique du Nord. Mémoires du Muséum National d'Histoire Naturelle, Série A, Zoologie, 11.

\section{Citation:}

Nourti Mohamed, Grichanov Igor Ya., Kettani Kawtar (2019). New records of long-legged flies (Diptera, Dolichopodidae) from Morocco. Acta Biologica Sibirica, 5 (3), 118-130.

Submitted: 10.07.2019. Accepted: 15.08.2019

cross ref http://dx.doi.org/10.14258/abs.v5.i3.6514

(C) 2019 by the authors. Submitted for possible open access publication under the terms and conditions of the

Creative Commons Attribution (CC BY) license (http://creativecommons.org/licenses/by/4.0/). 